\title{
A new moon for Neptune
}

\begin{abstract}
Hippocamp, a previously undetected moon of Neptune, has a peculiar location and a tiny size relative to the planet's other inner moons, which suggests a violent history for the region within 100,000 kilometres of the planet. SEE LETTER P.350
\end{abstract}

\section{ANNE J. VERBISCER}

$\mathrm{I}$ n 1989, the NASA spacecraft Voyager 2 detected six moons of Neptune that are interior to the orbit of the planet's largest moon, Triton ${ }^{1}$. On page 350, Showalter et al. ${ }^{2}$ report the discovery of a seventh inner moon, Hippocamp. Originally designated as S/2004 N 1 and Neptune XIV, this moon was found in images taken by NASA's Hubble Space Telescope in 2004-05 and 2009, and then confirmed in further images captured in 2016. Hippocamp is only 34 kilometres wide, which makes it diminutive compared with its larger siblings, and it orbits Neptune just inside the orbit of Proteus - the planet's second largest moon.

Discoveries in the past few years of moons and other objects that are associated with the outer planets of the Solar System have been serendipitous. For example, in 2012, astronomers found one of Neptune's trojans ${ }^{3}$ - bodies that share the orbit of a planet or other celestial body - while looking for a target for the NASA spacecraft New Horizons after its flyby mission to Pluto. And, in 2018, planetary scientists reported the discovery of 12 moons of Jupiter (see go.nature.com/2ge8ekk), which were identified during a search for the elusive Planet X that is proposed to lurk in the outermost region of the Solar System ${ }^{4}$.

By contrast, Showalter and colleagues were actively hunting for moons to add to Neptune's retinue. Showalter leads teams of researchers that specialize in finding small moons, using images from both space probes and satellites in orbit around Earth, including Hubble. In 1991, Showalter reported the discovery of $\mathrm{Pan}^{5}$, a small moon of Saturn that orbits in a gap in one of the planet's rings, by meticulously searching images taken nine years earlier by Voyager 2. Showalter's sleuthing has also netted small moons of Uranus (Mab and Cupid ${ }^{6}$ ) and Pluto (Kerberos and Styx ${ }^{7-9}$ ), using images from Hubble.

Many observation campaigns have focused Hubble's most powerful instruments on Neptune. Nevertheless, Hippocamp eluded detection until Showalter et al. implemented a specialized image-processing technique that enhanced the sensitivity of Hubble's cameras. This method effectively increases exposure times beyond the limit imposed by image

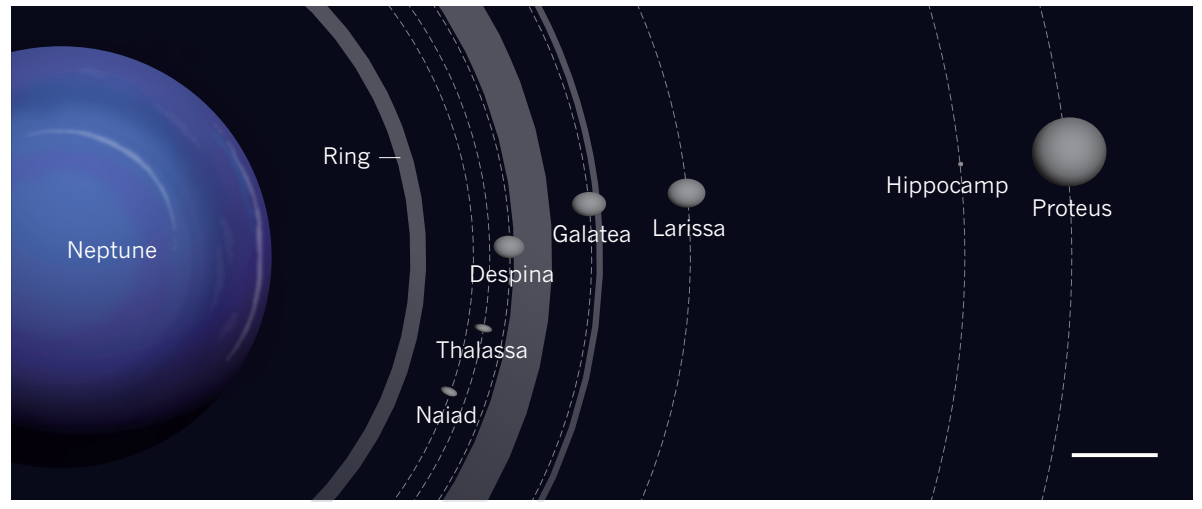

Figure 1 | The inner system of Neptune. Showalter et al. ${ }^{2}$ report the discovery of a moon of Neptune known as Hippocamp. Shown here is the location of Hippocamp, compared with those of Neptune's rings and other inner moons. The relative sizes of the moons are accurate; however, the moons have been enlarged with respect to their orbits by a factor of 20, to highlight the diminutive size of Hippocamp. Scale bar, 10,000 kilometres. (Adapted from Extended Data Fig. 4 of ref. 2.)

smearing that is caused by a moon's orbital motion.

Any moon with a circular orbit that is roughly aligned with a planet's equator and that moves in the same direction as the planet's rotation will follow a predictable path around the planet. As a result, sequential images can be transformed to match each other's appearance by relocating pixels in an original image to locations in subsequent images that coincide with the moon's orbital "The authors motion. Stacking the were actively transformed images hunting for extends exposure times from a few moonsto hundred seconds to Neptune's retinue." to tens of minutes, up to about $37 \mathrm{~min}$ utes. The application of a similar technique led to the discovery ${ }^{10}$ of $2014 \mathrm{MU}_{69}$ - an object in the outer Solar System that was visited by New Horizons in January this year (see Nature http://doi.org/ gfskgt; 2019).

Using the same procedure, Showalter and colleagues also observed Naiad - Neptune's innermost moon, which had not been seen since it was spotted by Voyager 2 in 1989. Following a comprehensive search using their image transformation and stacking technique, the authors concluded that there are no further moons larger than $24 \mathrm{~km}$ in diameter that are interior to the orbit of Proteus. Beyond
Proteus, 200,000 km away from Neptune, there are no moons wider than $20 \mathrm{~km}$.

The discovery of Hippocamp is intriguing because of the moon's relationship to Proteus and the role that both objects might have had in the history of Neptune's inner system. Hippocamp, the smallest known inner moon of Neptune, orbits just $12,000 \mathrm{~km}$ inside the orbit of Proteus, the planet's largest inner moon (Fig. 1). Both moons migrate outwards because of gravitational interactions with Neptune, but smaller Hippocamp moves much more slowly than Proteus. Therefore, Hippocamp resides nearer to the location at which it formed than does Proteus, which suggests that the two bodies were much closer together in the past.

Proteus sports an unusually large crater called Pharos - a telltale sign that the moon might have barely escaped destruction by impact. Whenever this impact occurred, it no doubt launched debris into orbit around Neptune. Although Showalter et al. propose that some of this debris coalesced to form Hippocamp, they do not explore the possibility that some of the dust remains today in the form of a ring, such as the gossamer rings of Jupiter $^{11}$ or the Phoebe ring of Saturn ${ }^{12}$.

Phoebe, an outer moon of Saturn, resides in - and is presumed to be the source of - the enormous dust ring that bears its name. Phoebe's own large crater, Jason, offers evidence of the moon's violent past, as does Pharos for Proteus. Intriguingly, although 
Showalter and colleagues note that the volume of Hippocamp is only $2 \%$ of that missing from the Pharos impact basin, the volume of particles in the Phoebe ring would fill a crater that is only $1 \mathrm{~km}$ wide ${ }^{12}$ (by comparison, Jason is about $100 \mathrm{~km}$ in diameter $\left.{ }^{13}\right)$. Clearly, the impacts that produced these revealing scars generated much more debris than remains today in the form of a dust ring or a tiny moon.

Whether Hippocamp formed in place from material that did not originate from Proteus or was born of Proteus remains to be determined.
Nevertheless, applying the techniques that were used to find it might result in the detection of other small moons around giant planets, or even planets that orbit distant stars.

Anne J. Verbiscer is in the Department of Astronomy, University of Virginia, Charlottesville, Virginia 22904, USA. e-mail:verbiscer@virginia.edu

1. Smith, B. A. et al. Science 246, 1422-1449 (1989)

2. Showalter, M. R., de Pater, I., Lissauer, J. J. \& French, R. S. Nature 566, 350-353 (2019).
3. Parker, A. H. et al. Astron. J. 145, 96 (2013).

4. Brown, M. E. \& Batygin, K. Astrophys. J. Lett. 824, L23 (2016).

5. Showalter, M. R. Nature 351, 709-713 (1991).

6. Showalter, M. R. \& Lissauer, J. J. IAU Circ. 8209 (2003).

7. Showalter, M. R. et al. IAU Circ. 9221 (2011).

8. Showalter, M. R. et al. IAU Circ. 9253 (2012).

9. Showalter, M. R. \& Hamilton, D. P. Nature $\mathbf{5 2 2}$ 45-49 (2015).

10.Spencer, J. R. et al. EPSC Abstr. 10, EPSC2015-417 (2015).

11.Burns, J. A. et al. Science 284, 1146-1150 (1999).

12. Verbiscer, A. J., Skrutskie, M. F. \& Hamilton, D. P. Nature 461, 1098-1100 (2009).

13. Giese, B., Neukum, G., Roatsch, T., Denk, T. \&

Porco, C. C. Planet. Space Sci. 54, 1156-1166 (2006).

\title{
How broken sleep harms blood vessels
}

\begin{abstract}
The link between sleep and cardiovascular disease is poorly understood. Findings in mice now show that disrupted sleep causes the brain to signal the bone marrow to boost white blood cell production, damaging blood vessels. SEE LETTER P.383
\end{abstract}

\section{ALAN R. TALL \& SANJA JELIC}

$\mathrm{M}$ ost people have at some point echoed Macbeth's complaint about the loss of "sleep that knits up the ravelled sleeve of care". Sleep disorders, such as obstructive sleep apnoea (when breathing temporarily stops, causing both sleep disruption and lack of oxygen in blood) and sleep deprivation, have been associated with an increased risk of atherosclerosis and its harmful cardiovascular effects ${ }^{1,2}$. Atherosclerosis is characterized by the formation of 'plaques' in arteries, as white blood cells enter the artery wall, take up cholesterol and other substances from the blood and trigger an inflammatory response. However, the mechanisms linking sleep disruption and atherosclerosis have been largely unknown. McAlpine et al. ${ }^{3}$ show on page 383 that persistent sleep disruption causes the brain to signal the bone marrow to increase the production of white blood cells.

McAlpine et al. studied mice that were prone to developing atherosclerosis. The authors induced sleep fragmentation by moving a bar intermittently across the bottom of the animals' cages during their sleep period (Fig. 1), and compared these animals with animals that slept normally. They found that mice with sleep fragmentation had moresevere atherosclerosis, which was paralleled by increases in the production of white blood cells in the bone marrow and in the numbers of monocytes and neutrophils - two types of white blood cell - in the blood. These effects were absent if the bar was moved when the mice were fully awake. Stress activates the sympathetic nervous system (which is associated with the 'fight-or-flight' response), and such activation increases the production of white blood cells and atherosclerosis in other experimental settings ${ }^{4}$. However, the authors did not find evidence for a role of sympathetic activation in their setting.

Next, McAlpine and colleagues performed a systematic examination of sleepregulating proteins in the hypothalamus, a part of the brain that controls metabolic responses in the body. They discovered that expression of the protein hypocretin (also called orexin) was markedly reduced in the sleep-disturbed mice, compared with the control mice. Hypocretin promotes wakefulness, and increases food intake and the activity of the sympathetic nervous system ${ }^{5}$. Low expression of hypocretin has been seen in individuals with narcolepsy, a rare disorder that makes people fall asleep during the day ${ }^{5}$.

McAlpine et al. observed that, like the mice with sleep fragmentation, genetically engineered mice unable to express hypocretin produced more white blood cells and had
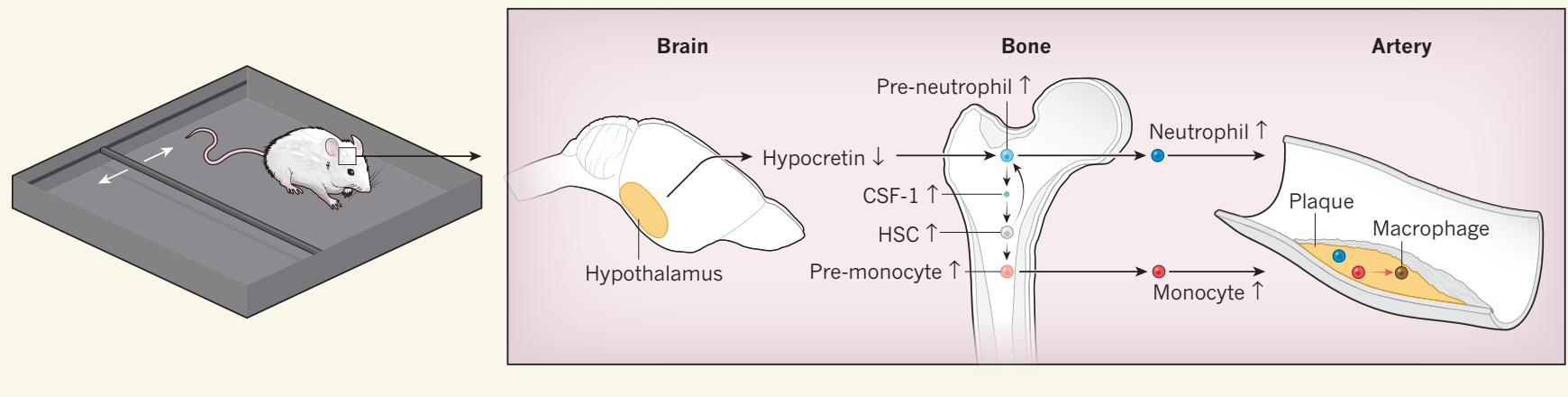

Figure 1 | Sleep fragmentation accelerates atherosclerosis. McAlpine et al. ${ }^{3}$ induced long-term sleep fragmentation in mice by moving a bar across the bottom of their cages during their sleep period. Such treatment reduces the levels of the protein hypocretin, which is produced in the brain's hypothalamus. Hypocretin receptors are present on cells in the bone marrow (pre-neutrophils) that will develop into a type of white blood cell called a neutrophil. Pre-neutrophils sense the lack of hypocretin and produce increased amounts of a protein known as colony-stimulating factor-1
(CSF-1). Uninhibited CSF-1 production stimulates stem cells that give rise to all types of white blood cell (haematopoietic stem cells; HSCs) to produce increased numbers of pre-neutrophils and pre-monocytes (white blood cells that will develop into monocytes). Mature neutrophils and monocytes migrate to arterial walls, where the monocytes develop into macrophage cells. Macrophages and, to a lesser extent, neutrophils promote the formation of plaques, a hallmark of atherosclerosis - a condition that can have harmful cardiovascular effects. 